\title{
AFRODESCENDIENTES EN COLOMBIA: UNA REVISIÓN DE LOS ÚLTIMOS SEIS AÑOS
}

\section{AFRICAN DESCENT IN COLOMBIA: A REVIEW OF THE PAST SIX YEAR}

\author{
Jhonatan C. Porto Sierra ${ }^{1}$
}

\begin{abstract}
RESUMEN
En el siguiente artículo se presentan una serie de estudios sobre la temática afrodescendiente, los cuales se han producido desde diversas disciplinas en Colombia desde el 2008 hasta el 2013. El objetivo principal de éste es exponer las publicaciones que se han elaborado después de la compilación bibliográfica publicada por Restrepo y Rojas (2008) hasta hoy. La realización de este artículo se basó en las fuentes disponibles sobre la temática como libros, artículos, tesis, etc., con los cuales se elabora una revisión de tipo cronológico en el lapso mencionado anteriormente. Se espera que, a través de esta publicación, se revelen contrastes sobre los estudios afro que se dan desde distintas perspectivas disciplinarias.
\end{abstract}

Palabras clave: Afrodescendiente, etnia, negro, esclavitud, etnicidad, racismo, afrocolombianos

\section{ABSTRACT}

The following a series of studies are presented on afro-descendant issues, which have been produced from various disciplines in Colombia from 2008 to 2013. The main purpose is to present the publications developed after the bibliographic compilation published by Restrepo and Rojas (2008) until today. This work is based on various sources such as books, articles, theses, etc. They made possible a chronological review of the researches produced during the abovementioned period. Through this publication, some contrasts on African studies will be revealed, these contrasts are given from different disciplinary perspectives.

Keywords: African Descent, ethnicity, black, slavery, ethnicity, racism, Afro-Colombians

Tipología: Artículo de revisión Fecha de recepción: 14/01/2014

Fecha de aceptación: 12/06/2014

Forma de citar este artículo: Porto, J. (2014). Afrodescendientes en Colombia: una revisión de los últimos seis años. Jangwa Pana, 13, 131 - 139

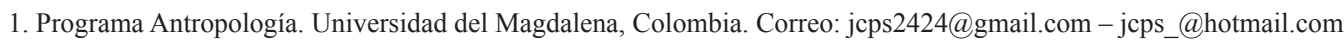




\section{INTRODUCCIÓN}

Sin duda, el legado africano en América, y es$S_{\text {pecíficamente en Colombia es tan grande que }}$ sólo pensar en negarlo puede resultar algo incomprensible o descabellado. Desde el siglo XVI, momento en el cual la esclavitud tuvo su mayor auge en toda la historia del territorio, en lo que actualmente constituye el territorio colombiano, se trajeron directamente desde África a América gran número de esclavos africanos, como explica Maya (1988) a través de una serie de estadísticas aportadas por Nicolás del Castillo (1982), solamente en el periodo que va desde 1533 a 1580 debieron llegar al territorio colombiano cerca de 3000 personas, las cuales, aportaron sin duda un enriquecimiento cultural muy amplio a un territorio cuya población era principalmente indígena. Las mezclas y tradiciones a las que hace referencia Del Castillo aún se presencian hoy en día; son las "huellas de africanía" que aún están presentes en el contexto colombiano y que sin importar el paso del tiempo han perdurado, dando como resultado un país que se proclama a sí mismo como multicultural. A pesar de lo anterior, la presencia de personas negras al momento de la realización de estudios académicos en la antropología, siempre ha estado relegada por la de los grupos indígenas, los cuales tienden a tener cierto protagonismo ante las personas afrocolombianas, tal como lo hace ver Walsh (2005), tanto en Colombia como en Ecuador, "la presencia afro ha sido históricamente invisibilizada especialmente al frente de lo indígena como paradigma central de análisis e interés académico" (Walsh, 2005: 02, citado en Restrepo \& Rojas, 2008: 03).

Dada la importancia que han tenido los distintos procesos históricos por los cuales ha pasado la población afro, y toda la importancia sociocultural que significa no sólo para Colombia sino

2. Concepto introducido por Nina S de Fridemann: 1997 "No obstante dicho concepto ha sido elaborado por Jaime Arocha desde la teorización de la formación de hábitos, recorriendo Gregory Batenson." (Friedemman, 1997: 175, citado en, Restrepo, 2003: 92) A demás el término fue renovado por el análisis histórico aportado por Adriana Maya, es decir que el término es un aporte en conjunto de los tres autores. para todo el continente americano, en los últimos tiempos (desde la década de 1950 con Escalante, Arboleda y Price) lo referente al tema afro ha despertado un interés muy grande en el ámbito académico, como es el caso de autores más contemporáneos como Restrepo (2003, 2009), Moreno (2011), Wade (2002), entre otros.

Este artículo no pretende exponer todos los trabajos que se han producido desde la comunidad académica hasta hoy, sino mostrar una serie de trabajos realizados en los últimos seis años. Se toma este punto de partida, ya que Restrepo ha realizado compilaciones bibliográficas muy completas con respecto a este tema en años anteriores (1999-2008). La primera fue el resultado de una revisión bibliográfica inicial para su trabajo de grado, la cual fue publicada el 1999 bajo el nombre de "Poblaciones negras en Colombia: compilación bibliográfica", dando como resultado un hipertexto como él mismo lo denomina, que así mismo remite a otros textos de interés en la temática afro. En el 2008, realizó una segunda publicación titulada "Afrodescendientes en Colombia: una compilación bibliográfica" con la colaboración de Axel Rojas ${ }^{3}$, donde hacen una exhaustiva revisión agrupando más de 500 publicaciones, tanto impresas como digitales. Restrepo y Rojas (2008) explican la importancia de tener en cuenta todos los referentes que se han producido sobre la población afrodescendiente, aclarando que ninguna publicación es más importante que otra, que su valor depende del lector que lo tenga a su disposición y de lo que se quiere encontrar. Los autores (Restrepo y Rojas, 2008) reconocen la probabilidad de que su trabajo no esté completo, ya que la bibliografía en temas afro es muy vasta por lo cual de manera autocrítica, son conscientes de que puede tener ciertos vacíos.

El campo de acción de este trabajo está enmarcado en la antropología social, pero esto no quiere decir que trabajos de tipo histórico, sociológico

3. Profesor de la Universidad del Cauca. Su área de interés está centrada en estudios afrocolombianos, multiculturalismo y políticas educativas. 
e incluso de tipo político o de cualquier otra área de conocimiento relacionada con el tema de interés no será tenido en cuenta, ya que considero que cualquier producción de conocimiento es importante para comprender la realidad de estas poblaciones al igual que Restrepo y Rojas (2008), concuerdo con la idea de que toda producción bibliográfica es importante, que su valor es directamente proporcional al tema de interés de cada lector. De este modo, lo que realmente se quiere es, presentar las publicaciones más relevantes en la temática afrodescendiente que se han dado en Colombia desde el 2008, año en que fue publicada la compilación bibliográfica de Restrepo y Rojas hasta hoy en día, exponiendo distintos trabajos e integrándolos entre sí, con el fin de establecer comparaciones, relaciones e incluso contrastes que se den en éstas.

La metodología que se empleó fue la revisión bibliográfica en revistas, bibliotecas, artículos, documentos de instituciones del Estado y demás material bibliográfico tanto impreso como magnético.

\section{Un vistazo desde distintas disciplinas}

Un buen punto de partida es el trabajo realizado por Wade, Urrea y Viveros (2008) "Raza, etnicidad y sexualidades. Ciudadanía y multiculturalismo en América Latina", éste es el resultado de dos seminarios realizados en el Reino Unido y en Colombia entre el 2006 y el 2007, donde los tres autores hacen una recopilación de los temas tratados en el seminario; todos los temas tratados ven la relación que existe entre la discriminación racial y la discriminación sexual en el contexto afro de distintos países latinoamericanos y de Europa, donde la violencia y el racismos son eminentes. García (2012) también muestra las problemáticas que enfrentan las personas afro en la ciudad de Medellín, siendo protagonista de escenarios de discriminación y violencia, que imposibilitan el pleno desarrollo de estas personas, propiciando de esta manera, "el atraso y la marginalidad" que viven las personas afrodescendientes de esta ciu- dad, siendo la violencia un factor común entre estos dos trabajos. El trabajo de Hernández (2009), el cual es una producción de tipo histórico, que relata cómo ha sido la resistencia civil del país en poblaciones afro, campesinas e indígenas en las dos últimas décadas del siglo XX, producto del conflicto armado, que trae como gran consecuencia el desplazamiento; este texto abarca tres departamentos, cada uno muestra una presencia significativa de los grupos étnicos en cuestión, Santander (campesinos), el Cauca (indígenas) y Chocó (afros) ilustrando qué sucede en cada uno. El Chocó es uno de los departamentos en los que más población afro vulnerable hay en el país, esto se puede apreciar principalmente en tres trabajos; el primero de ellos es el de Bustamante (2011) donde se expone a través de estadísticas hechas por el $\mathrm{DANE}^{4}$, que el Chocó en el 2011 fue el departamento número uno en cifras relacionadas con la pobreza, con el 64,0\%; a pesar de que este trabajo hace un aporte importante en lo referente a estadísticas, se queda corto al momento de exponer las razones por las cuales el índice de pobreza en este departamento es tan alto. Sin embargo, al recurrir al artículo del Observatorio del programa presidencial de derechos humanos y DIH (2009), en este punto se aclara un poco, pues muestra cómo el Chocó, a pesar de ser un departamento muy rico, sigue viviendo en la pobreza, donde sus principales actividades económicas son la extracción de oro y plata, y la explotación del bosque forestal; pero aun así no logra superar esta pobreza porque los recursos son usados por empresas multinacionales que los extraen sin retribuir nada a la comunidad y siendo esto una de las principales causas de su pobreza. Así mismo, esto se confirma con el artículo de Leal (2009) quién hace un barrido histórico entre el período de 1987 a 1930, y narra cómo el país era el principal exportador de platino del mundo, siendo exportado en su mayoría por la compañía minera Chocó Pacífico en el río Condoto sin que el país haya recibido regalías por extracción de

4. El Departamento Administrativo Nacional de Estadística (DANE), es la entidad responsable de la planeación, levantamiento, procesamiento, análisis y difusión de las estadísticas oficiales de Colombia. 
ese metal, mostrando cómo el estado de pobreza de este departamento no es nuevo, ya que viene de un proceso histórico largo, y genera, como consecuencia, el estado actual en que se encuentra el Chocó.

Así mismo, Restrepo (2013) relata que las ideas de que el Pacífico es un territorio que posee una biodiversidad muy grande, no es nueva y que no surge de un día para otro, más que esto, ver el Pacífico en términos de su riqueza natural está anclado a procesos históricos que muchas veces no se tienen en cuenta, esto es porque la idea de su biodiversidad nos parece tan obvia que se ha impregnado a nuestro imaginario social y político, dándola por sentado pero sin detenernos a analizarla. Las ideas a las que hace referencia están dadas, en primer lugar, por una serie de imágenes originadas en el período colonial y que siguen impresas en la imagen que se tiene del Pacífico; por ejemplo, que su entorno está rodeado de tierras incultas, grandes selvas, animales salvajes, torrenciales lluvias, etc., teniendo en cuenta que estas imágenes fueron dadas aportadas principalmente por viajeros y exploradores que llegaron a la región. Como segundo aspecto, se tiene la biodiversidad de este territorio, del Pacífico que no es algo que sale, de repente a la luz, más bien, produce una serie de "inteligibilidad y legibilidad" (Restrepo, 2013) porque los discursos sobre la biodiversidad del Pacífico se crearon en base a la gran flora y fauna que se encuentra en la región, esto en un primer plano, porque más adelante (primera década de los 90's) se vería la misma biodiversidad pero en términos de riqueza y que crearían la posibilidad de que Colombia estuviera ubicada dentro de los parámetros desarrollistas de la época. Es decir, que separa de la idea de ver a la región no tanto por su valor netamente natural, sino que va atado a propósitos capitalistas que buscan un incremento a nivel monetario.

Los estudios en temática afro del país en los últimos años, también han estado muy relacionados con el tema de la educación en las minorías étnicas, entre los estudios relacionados en el campo pedagógico, es importante resaltar el papel de la Cátedra Estudios Afrocolombianos, la cual se creó a través de la Ley 70 de 1993, donde se hace necesario que en Colombia se establezcan estudios que vayan acorde con las personas pertenecientes a minorías étnicas como indígenas y negros. Para llevar a cabo esta labor, se hace necesario implementar una etnoeducación, la cual "debemos entenderla como la educación en los valores de la etnicidad nacional, teniendo en cuenta que nuestra identidad cultural es el sincretismo o mestizaje de tres grandes raíces: la africanidad, la indigenidad y la hispanidad" (Mosquera, 1999). Un buen ejemplo de esto es el trabajo de Lamus (2010) titulado "San Basilio de Palenque siglo XXI: Lengia ri palenge y Proyecto Etnoeducativo" donde se expone el caso de un grupo de mujeres palenqueras quienes, a través de la etnoeducación, refuerzan su lengua natal y demás costumbres propias de su cultura, con la cátedra afrocolombiana que se dicta en el Instituto Benkos Biohó, lo cual dio como resultado, una reafirmación de las tradiciones propias de San Basilio como la venta de dulces, enseñanza de la lengua, fortalecimiento económico, etc. Otro buen ejemplo es el expuesto por Moreno (2011), donde se hace un análisis histórico de la comunidad afrocolombiana, con un énfasis en conceptos claves para comprender el tema étnico, los elementos históricos que han moldeado de alguna manera a la población afrocolombiana, y así mismo ver su estado actual; esta publicación, es un módulo dirigido a mujeres afro, que posibilitará conocer de una manera didáctica más sobre su cultura. El tema de la etnoeducación ha tenido una gran acogida en poblaciones afro, y así mismo ha motivado a que distintas organizaciones trabajen con el Estado en ayudar a estas poblaciones; como el caso del Ministerio de Cultura y El Observatorio del Caribe, que lideraron el proyecto para la salvaguardia de la lengua palenquera (2012), donde se implementó la etnoeducación para el fortalecimiento de la lengua en San Basilio de Palenque, que está inmersa en su historia, prácticas, cosmovisiones y tradición oral; la cual requiere para su rehabilitación, acciones 
de uso, apropiación, transmisión y difusión; ya que constituye un símbolo de resistencia que refuerza la integración social y re-crea la memoria de San Basilio.

Las temáticas afro cubren un espectro muy amplio, en esta revisión se han encontrado estudios en campos como la salud, donde se detectan patologías que están relacionadas muy de cerca con el estilo de vida de las personas. Las enfermedades de transmisión sexual, son muy comunes en esta población debido a vulnerabilidad tanto étnica como social y económica, como lo exponen Arrivillaga y colaboradores (2011) dando a conocer la situación que se vive en el Pacífico en relación al VIH, el cual está presente en gran parte de la población, sobretodo en personas mayores a los 35 años de edad, atribuyéndole distintas causas a este problema como el bajo nivel de educación, la violencia, la pobreza, y la vulnerabilidad, entre otros. Así mismo hacen un aporte valioso al mostrar otras enfermedades muy comunes en esta región, por ejemplo: paludismo, fiebre tifoidea, cáncer, infecciones respiratorias, desnutrición etc.

La medicina tradicional de la cultura afrodescendiente también forma parte de este campo de estudio de la salud, y se puede apreciar en el trabajo hecho por Argote, Mejía, Vásquez y Villaquirán (2008) donde, a través de una muestra de 17 mujeres, evidencian la relación que las mujeres tienen con su propio cuerpo, sus defensas y fortificación desde la menarquía ${ }^{5}$ como fase preparatoria de sus funciones reproductivas, hasta el momento de la menopausia, ya que de acuerdo con la cultura, hay ciertas prácticas que, de no ser tenidas en cuenta, podrían causar diversas dolencias en la etapa final de la procreación, sobre todo en los cuidados referentes al significado de la sangre, el ejercicio de la sexualidad, limpieza, alimentación especial, el equilibrio que debe haber entre el calor y el frío, etc. De un modo muy similar, Restrepo, Arboleda, Ramírez y Álvarez

5. Es el día en el cuál se produce el primer episodio de sangrado vaginal de origen menstrual, o primera hemorragia menstrual de la mujer
(2011) exponen cómo las poblaciones afrodescendiente y mestizas de los departamentos de Chocó y Antioquia, contraen dengue hemorrágico, donde la principal causa radica en la calidad de vida y las condiciones en las que viven las personas, donde no hay buena salubridad, razón principal por la que se contrae esta enfermedad, lo cual a su vez deteriora aún más el nivel de vida de las personas; se encontró que la población afro es más propensa a contraer esta enfermedad, en comparación con la población mestiza de Antioquia. Lo expuesto anteriormente va acorde con lo que muestra Asprilla (2009) quien a través de la encuesta como herramienta metodológica, revela las condiciones en las que viven muchas personas pertenecientes a la población afro del país. Asprilla (2009) muestra que las personas afro no necesariamente carecen de ingresos monetarios; lo que en realidad es propio de esta población, es que dichos ingresos no son suficientes para mantener buenas condiciones de salubridad, por lo que siempre tienden a estar más expuestas a escenarios que atentan contra su salud, siendo el desplazamiento la principal causa de todo esto. El desplazamiento en la población afro, es un tema al cual se le han dedicado varias publicaciones entre las cuales vale la pena destacar el libro elaborado por Rodríguez, Alfonso y Cavelier (2009) donde ilustran las causas, consecuencias y peligros a los que están sujetos las personas que son víctimas del desplazamiento forzado en el país; es muy relevante porque no sólo exponen estadísticas acerca del desplazamiento, sino que además van explicando de manera continua dichas estadísticas, haciendo ver diversos datos de interés en lo referente a esta problemática, por ejemplo: muestran que el principal motivo del desplazamiento son los desastres naturales que se presentan en las zonas rurales donde viven generalmente estas personas, seguido de amenazas para la vida por parte de grupos armados y por motivos de salud; también exponen el alto índice de población afrodescendiente que es víctima del desplazamiento, que "de hecho representan casi la cuarta parte de la población desplazada del país (22,5\%)" (Rodríguez, C., Alfonso T., \& 
Cavelier, I., 2009) lo cual es muy grave teniendo en cuenta que según estos mismos autores los afrodescendientes son la minoría étnica más grande del país y así mismo la más grande en número de desplazados, seguida por la población indígena y campesina. Además de lo anterior, continúan exponiendo más aspectos del desplazamiento, revelando que la mayor concentración de desplazados afro del país se encuentra en el Pacífico, lo cual se puede ver en otras publicaciones como la realizada por un grupo de investigadores de CODHES$^{6}$ (2013) donde se analizan las tasas de desplazamiento en el 2012 que se dieron en distintos departamentos del país, y donde arroja como conclusión que en el Pacífico, es donde más desplazamiento se presenta en Colombia, donde las personas huyen de sus viviendas principalmente por las confrontaciones entre grupos armados, homicidios, hostilidades, amenazas provocadas por las $\mathrm{FARC}^{7}, \mathrm{ELN}^{8}$ y la Fuerza Pública, expulsando a municipios como Tadó, Medio de San Juan, Ríosucio Medio Baudí, dando como resultado "7.703 víctimas, de las cuales 2.911 fueron indígenas y 3.592 fueron afrocolombianos" (Londoño, et. al, 2013).

La población más afectada por el desplazamiento son las mujeres, Albán (2012) lo expone a través de una reseña elaborada al libro de Doris Lamus "El color negro de la (sin) razón blanca: El lugar de las mujeres afrodescendientes en los procesos organizativos en Colombia." En este libro se ilustra la situación actual de las mujeres por reivindicar sus derechos y posición social en el país, sobretodo en el Pacífico y en el Caribe colombiano, la cual siempre ha estado atada a nociones de discriminación que van de cerca a procesos históricos enmarcados así mismos a procesos capitalistas, que obedecen a lógicas eurocéntricas atadas a nociones como raza, etnia, racismo, etc., siguiendo la continuidad y la necesidad que se ha creado de establecer una relación de superior/inferior, "en este sentido, tanto raza como etnia van

6. Consultoría para los derechos humanos y desplazamiento 7. Fuerzas Armadas Revolucionarias de Colombia

8. Ejército de Liberación Nacional a denotar una construcción social que identifica tanto la diferencia como la igualdad." (Albán, 2012) Así mismo, continúa el debate en torno a las relaciones de género como una categoría crítica y política, cuyos usos la han enmarcado en debates de desarrollo, mostrando así que las relaciones de género son un tema de poder $\mathrm{y}$, por lo tanto deben ser tratados en contextos específicos, que obedezcan a las dinámicas del grupo en que se esté.

Así mismo Bernal (2010) ilustra esta situación mostrando en su artículo algunos apartes referentes a la constitución y los derechos que provee a la población afro, pero que así mismo deja por fuera muchas cosas relacionadas al género, equidad de género y derechos humanos de la población afrocolombiana y de las demás minorías étnicas del país; según la autora, son las mujeres y las niñas quienes constituyen la mayor población desplazada del país, exponiendo que no es una mera coincidencia referente a su condición sexual, sino que va más allá, atada a problemáticas sociales más profundas, ya que ellas son por lo general las cabezas de la familia, las encargadas del hogar y del cuidado de los hijos, viéndose obligadas a huir cuando están expuestas a situaciones de riesgo. En el 2013, se presenta el "Informe Sombra al Comité para la Eliminación de la Discriminación contra la mujer" en el marco de la Convención sobre la Eliminación de todas las formas de Discriminación contra la Mujer CEDAW, elaborado por el kuagro Ri Ma Changaina Ri PCN (colectivo de mujeres PCN), donde se exponen las principales preocupaciones en lo referente a la vulnerabilidad de los derechos tanto individuales como colectivos de la mujer, así mismo de la alta vulnerabilidad y discriminación que hay en contra de ésta. Un punto importante que se discute es la falta de pertenencia tanto étnico cultural y de género en políticas y recursos legislativos por parte del Estado, y que afectan directamente a las personas vulnerables porque no poseen un medio para defender sus derechos. En este informe se apela a peticiones en lo referente a consulta previa, inclusión de géne- 
ro, un plan de Derechos Humanos y de Derecho Internacional Humanitario que vaya acorde a la población negra del país, realizando estudios que muestren la verdadera situación que viven las mujeres afrodescendientes que, infortunadamente, está ligado a discriminación y violencia, ya sea por género, raza, procesos de exclusión, violencia, desplazamiento, etc.

En el campo psicológico la ayuda es necesaria en términos de trastornos que sufren las personas que viven en los escenarios de violencia constante, como lo describen Londoño y colaboradores $\left(2005^{\circ}\right)$ quienes al estudiar a Bojayá (Chocó) encuentran que las personas que son vulneradas, tienden a padecer de síntomas de ansiedad y de trastornos del estado de ánimo, trastorno de estrés postraumático, aumentando el índice de suicidios en una cantidad considerable, dado que las ganas de salir del estado violento al que se enfrentan a diario no les permite vivir plenamente. Aranguren (2009) tomando como referente las formas violentas en que se silencia, se extermina y se margina a las personas, ilustra las consecuencias que tienen para desarrollarse después en su vida diaria, los temores, abusos a los que son sometidos, los persiguen y son un recordatorio constante de los que les sucedió, imposibilitando en muchos casos, continuar con su vida.

\section{A manera de reflexión}

Lo expuesto en los párrafos anteriores ilustra cómo las personas afrocolombianas han estado atadas siempre a situaciones de violencia, pobreza, marginalidad y demás escenarios que no les ha permitido, en muchos casos, poder acceder a una vida digna, tanto en términos de estabilidad económica como emocional y de seguridad social, etc. Además, se puede apreciar cómo la gran mayoría de los estudios afro realizados Colombia tienen como denominador común a la violencia, tanto en temáticas psicológicas, educativas, médicas, antropológicas e históricas que muestran

9. A pesar de no corresponder a los años fijados en esta compilación (20082013), es pertinente exponerlo dado el aporte que hacen en la temática. que esta situación viene sujeta a un largo proceso histórico, desde los procesos de colonización, donde la presencia del negro fue invisibilizada, ligada a abusos, maltratos, rechazo por su identidad, etc., y que esto, desafortunadamente sigue latente en el país. Sobre este tema tan controversial, podemos concluir que la invisibilización y el rechazo a la comunidad es una realidad, que se puede encontrar en escenarios como el Pacífico colombiano, donde está la mayor cantidad de personas afrodescendientes vulnerables, las cuales reciben abusos constantemente por parte de grupos al margen de la ley y de multinacionales que no le reconocen que ese es su territorio y por lo tanto el oro o cualquier otro metal precioso que se encuentre en este lugar les pertenece y que si se va a explotar, lo mínimo que se podría hacer es llegar a un acuerdo con la comunidad, a través de una consulta previa que revele cuáles son sus necesidades y qué se necesita realmente en el lugar; además de tener en cuenta que más que una obligación ésta, es un derecho fundamental que tienen los grupos étnicos, que protegen, a través de medidas legislativas la integridad, valores, y entereza cultural, social y económica con el derecho a participar de las comunidades.

En este orden de ideas, también se espera que a través de esta pequeña compilación se haga un aporte a las investigaciones en temáticas afro en Colombia tanto para la comunidad científica como para las mismas personas pertenecientes tanto a ésta, como a cualquier otra minoría étnica que son víctimas de los abusos constantes, para que se informen y tengan conocimiento respecto a sus derechos y alternativas para superar estos percances como estrategias como la etnoeducación, conocimiento de sus derechos contemplados en la Constitución política, estrategias de fortalecimiento en relación a sus valores y tradiciones que re afirmen y protejan esa ancestralidad. De igual forma, cabe enfatizar la importancia de los estudios afro para el país, y específicamente a la Costa Caribe, de invitar a la comunidad científica a que continúe investigando e indagando sobre este ámbito y, así mismo, a 
que seamos un poco más críticos sobre la situación por la que vive esta comunidad actualmente, darnos cuenta que a pesar de que la población afro siempre se toma como un punto referente a exclusión o violencia, esto no es lo único que la compone, sus gentes y sus tradiciones, el legado histórico que hay detrás de ellos, y los procesos actuales de reintegración social, corresponden a distintas realidades, que nos hacen ver que no hay una historia única; sino, que por el contrario hay mucho más allá de lo que se nos ha contado, de lo que hemos leído, de que sí, efectivamente existe violencia, pero más allá de eso, hay personas que cada día luchan por pertenecer a una sociedad donde no haya discriminación de ningún tipo, llámese de género, racial, condición social, niveles de educación, etc.

\section{AGRADECIMIENTOS}

Especialmente quiero agradecer a Dra. Claudia Rojas-Sepúlveda, quien con paciencia y dedicación me llevó, a través de sus consejos y asesorías a la realización de este artículo. Sin ella, nada de esto hubiera sido posible.

138 Así mismo, a los profesores Álvaro Acevedo y Tony de la Cruz los cuales, a través de sus respectivas áreas de investigación llevaron a despertar mi interés por la temática afro.

\section{REFERENCIAS BIBLIOGRÁFICAS}

Albán, A. (2012). Reseña de "El negro de la (sin) razón blanca: El lugar de la mujeres afrodescendientes en los procesos organizativos en Colombia" de Doris Lamus Canavate. Reflexión Política, 14, (27): 178-182. Aranguren, J. (2009). Subjetividades al límite: los bordes de una psicología social crítica. Universistas Psychologica, 8 (3): 601-613.

Argote, L., M. Mejía, M. Vásquez \& Villaquirán de González M. (2008). Climaterio y menopausia en mujeres afrodescendientes: una aproximación al cuidado desde su cultura. Aquichán, Vol. 8, núm. 1, pp. 33-49.
Arrivillaga, M., D. Correa, L. Tovar, H. Zapata, M. Varela, \& Hoyos, P. (2011). Infecciones de transmisión sexual en la región Pacífica colombiana: implicaciones para población en situación de vulnerabilidad étnica, social y económica. Pensamiento Psicológico, IX (16), 145-152.

Asprilla, M. (2009). Hogares afrocolombianos: un análisis indicativo de la pobreza y de la vulnerabilidad social a partir de la encuesta de calidad de vida 2003. Tesis de grado para optar por una maestría en política social. Pontificia Universidad Javeriana. Bogotá-Colombia. Pp. 137.

Bernal, D. (2010). Desplazamiento forzoso y derechos de las mujeres pertenecientes a comunidades afrodescendientes en Colombia. Universidad Republicana Vol. 8: 71-80. Bustamante, J. (2011). Pobreza monetaria por departamentos. Oficina de Prensa DANE. Recuperado de: http://www.dane.gov.co/files/ investigaciones/condiciones_vida/pobreza/cp_ pobreza_2011.pdf

Consultoría para los Derechos humanos y Desplazamiento CODHES. (2013). La crisis humanitaria en Colombia persiste, El Pacífico en disputa: informe de desplazamiento forzado en 2012. Documento CODHES N 26. Recuperado de: http://www.lwfcolombia. org.co/sites/default/files/image/310513\%20 Informe $\% 20 \% 20$ desplazamiento $\% 202012$.pdf Convención sobre la eliminación de todas las formas de eliminación de discriminación contra la mujer CEDAW. (2013). Informe sombra al Comité para la eliminación de la Discriminación contra la Mujer. Recuperado de: http://www. afrocolombians.com/pdfs/COLOMBIAInformeSombra-KuagroPCNorg.pdf

García, A. (2012). Espacialidades del destierro y la re-existencia. Afrodescendientes desterrados en Medellín, Colombia. Universidad de Antioquia. La Carreta Editores E.U. Pp. 168.

Hernández, E. (2009). Resistencia para la paz en Colombia. Experiencias indígenas, afrodescendientes y campesinas. Revista de Paz y Conflictos, No 2, pp. 117-135. 
Lamus, D. (2010). San Basilio de Palenque siglo XXI: Lengia ri palenge y Proyecto Etnoeducativo. Reflexión Política, 12 (24). Pp. 86-99.

Leal, C. (2009). La compañía Minera Chocó Pacífico y el auge del platino en Colombia, 1897-1930. Artículo de Edición especial, Universidad de los Andes- Colombia. Pp. 150-164.

Londoño H., O, Muñiz, J. Correa, C. Patiño, G. Jaramillo, J. Raigoza, L. Toro, D. Restrepo, \& C. Rojas (2005). Salud mental en víctimas de la violencia armada en Bojayá (Chocó, Colombia). Revista colombiana de psiquiatría, vol. XXXIV. Núm. 4, pp. 493-505.

Maya, L. (1988). Demografía Histórica de la Trata por Cartagena 1533-1810. Facultad de Ciencias Sociales Universidad de los Andes. Publicación digital en la página web de la Biblioteca Luis Ángel Arango del Banco de la República <http://www.banrepcultural. org/blaavirtual/geografia/afro/demografia maya\#Togoville $>$ Búsqueda realizada el 17 de marzo de 2014.

Ministerio de Cultura y Observatorio del Caribe. (2012). Plan especial de salvaguardia de la lengua palenquera. Recuperado de: https://www. google.com.co/url? sa $=\mathrm{t} \& \mathrm{rct}=\mathrm{j} \& \mathrm{q}=\&$ esrc $=\mathrm{s} \& \mathrm{~s}$ ource $=$ web $\& c d=1 \&$ ved $=0 \mathrm{CCkQFjAA} \& u r l=\mathrm{ht}$ tp $\% 3 \mathrm{~A} \% 2 \mathrm{~F} \% 2 \mathrm{Fwww}$.ocaribe.org\%2Fcargar imagen.php\%3Ftipo\%3D22\%26id\%3D22\& ei=noOCUuCJLIm2kQek1YDgDQ\&usg=A FQjCNGWFrNsEK-BC1ivhEyJdTwyHU_ aBw\&sig2=brk-gjMe4f6VBcc8ZUqnmg Cartagena- Colombia.

Moreno, P. (2011). Afrocolombianos: La Historia De Nuestro Futuro. Publicado en: http://www. manosvisibles.org. Bogotá-Colombia.

Mosquera, J. (1999). La etnoeducación y los estudios afrocolombianos en el sistema escolar. Santa Fe de Bogotá: Docentes Editoriales. Publicación digital en la página web de la Biblioteca Luis Ángel Arango del Banco de la República $<$ http://www.banrepcultural.org/blaavirtual/sociologia/cimarron/indice.htm>
Observatorio del programa presidencial de derechos humanos y DIH. (2009). Diagnóstico de la situación de los municipios habitados por las comunidades afrocolombianas priorizadas por la Honorable Corte Constitucional en el departamento del Chocó. Agenda presidencial para la Acción Social y la cooperación internacional. Pp. 65.

Restrepo, E. (1999). Poblaciones negras en Colombia: compilación bibliográfica. Documento de trabajo número 43. Facultad de ciencia sociales y económicas de la Universidad del Valle. Pp. 63. En: http://bibliotecavirtual.clacso.org.ar/ar/libros/colombia/cidse/doc43.pdf.

Restrepo, E. (2003). Entre Arácnidas, Deidades y Leones Africanos: Contribución Al Debate Afroamericanista En Colombia.Tabula Rasa: Revista De Humanidades. Vol. 1: 87-123

Restrepo,E\&A.Rojas,(2008).Afrodescendientes en Colombia: una compilación bibliográfica. Instituto de Estudios Sociales y Culturales, Pensar. Universidad del Cauca. Editorial Universidad del Cauca. Pp. 105

Restrepo, E. (2013). El giro a la biodiversidad en la imaginación del Pacífico colombiano. Revista Estudios del Pacífico Colombiano No.1:171-199.

Restrepo, B., M. Arboleda, R.Ramírez, \& G. Álvarez, (2011). Actividad sérica de la acetilhidrolasa del factor activador de plaquetas en pacientes afrodescendientes y mestizos con dengue, Colombia. Instituto Colombiano de Medicina Tropical-Universidad CES, Medellpin, Colombia.

Rodríguez, C., T. Alfonso, \& I. Cavelier (2009). El desplazamiento afro: tierra violencia y derechos de las comunidades negras en Colombia. Universidad de los Andes, Facultad de Derecho, Centro de investigaciones sociojurídicas. Colección Estudios CIJUS. Pp. 274.

Wade, P., F. Urrea, \& M.Viveros (2008). Raza, etnicidad y sexualidades. Ciudadanía y multiculturalismo en América Latina. Universidad del Valle: Centro de Investigaciones y Documentación Socioeconómica. Facultad de Ciencias Sociales y Económicas. Pp. 565. 